\title{
Análisis métrico de las comunicaciones de la revista Centro Azúcar
}

\author{
Luis Ernesto Paz Enrique \\ Alejandro Céspedes Villegas \\ Eduardo Alejandro Hernández Alfonso \\ Universidad Central "Marta Abreu" de Las Villas - UCLV, Cuba
}

ARTICLE

\begin{abstract}
Resumen
Las publicaciones científicas en línea generalmente se apoyan en los análisis métricos, favoreciendo la descripción de las revistas para su mejora continua. La revista Centro Azúcar se encuentra hace varios años en evaluación por bases de datos de prestigio internacional. La publicación carece de un estudio métrico que refleje la calidad de la publicación en consonancia a los indicadores establecidos por las fuentes de indización. Se plantean como objetivos del estudio examinar la producción científica de la revista Centro Azúcar desde el 2011 hasta el 2015, examinar las referencias contenidas en el período mencionado e identificar los índices de endogamia editorial presentes en la productividad y las referencias de los artículos publicados por la revista. Para la obtención de resultados se emplean métodos en los niveles teórico y empírico, destacándose el método bibliométrico. Se identifican varios aspectos que atentan contra la calidad de la publicación. Se aborda de forma novedosa el comportamiento de la endogamia editorial en las referencias. Se emiten valoraciones y se establecen aspectos a considerar por la publicación para que sea indizada en bases de datos de prestigio internacional.
\end{abstract}

Palabras clave

Estudio bibliométrico ; Publicaciones seriadas en línea ; Producción científica; Referencias bibliográficas ; Endogamia editorial ; Revista Centro Azúcar ; Cuba

\section{Metric analysis of communications of the journal Centro Azucar}

\begin{abstract}
Online scientific publications, generally suport on metric analysis, favoring the description of the journals for continuous improvement. The journal Centro Azúcar has been in evaluation bye databases of international prestige for several years. The publication lacks a metric study that reflects the quality of the online publication with the indicators established by indexing sources. It is presented as objectives of the study to examine the scientific production of the Centro Azúcar journal from 2011 to 2015, examining the references in the mentioned period and identify the editorial endogamy indices present in productivity and references of the articles published by the journal. To obtain results are used methods in the theoretical and empirical levels, standing out bibliometric method. Are identified several aspects that attempt against the quality of the publication. Is aborded in new form the editorial endogamy in the references of the articles published. In the study are mentioned aspects to consider for the publication, to be indexed in databases of international prestige.
\end{abstract}

Keywords

Bibliometric study; Online serials publications; Scientific production; Bibliographic references ; Editorial endogamy; Centro Azúcar Journal ; Cuba 


\section{Introducción}

La bibliometría es la especialidad métrica que analiza repertorios bibliográficos para facilitar la toma de decisiones. Los estudios métricos de la información aplican métodos y modelos matemáticos a unidades de información. La bibliometría es la disciplina que se encarga de analizar repertorios bibliográficos. A partir del surgimiento de la bibliometría se desarrollaron otras disciplinas métricas como la informetría, biblioteconometría, archivonometría y más recientemente la cibermetría y webmetría. A decir de Miguel \& Dimitri (2013): los estudios métricos contribuyen a describir el comportamiento de una variable de la ciencia. Puede utilizarse este método para reflejar la evolución de una publicación como lo muestran los estudios desarrollados por Bemabeu et al., 2012; Herculano, 2015; Paz et al., 2015; Olmeda \& de Moya, 2016; García et al., 2016; González y Osca, 2016.

La evaluación de la actividad científica en las distintas áreas del conocimiento está directamente relacionada con el desarrollo de las mismas. A decir de Paz \& Hernández (2015): “la producción de la ciencia tiene un carácter exponencial. Cada año aumenta el número de publicaciones, la cantidad de revistas científicas y los investigadores que se posicionan en el canal de la comunicación científica". Este fenómeno se describe de forma cíclica donde cada autor que publique tiene la posibilidad de ser citado. Este factor disminuye al existir mayor cantidad de publicaciones. Los autores generalmente procuran publicar en revistas de alto impacto, indizadas en importantes bases de datos internacionales. El hecho de publicar en estas revistas no significa que el autor sea citado, sin embargo hay una alta probabilidad que así sea. Los investigadores a su vez referencian revistas de alto impacto garantizando que su publicación cuente con actualidad y con un marco referencial de prestigio.

La Bibliometría es la ciencia que estudia la naturaleza y curso de una disciplina (en tanto en cuanto que dé lugar a publicaciones), por medio del cómputo y análisis de las varias facetas de la comunicación científica escrita. Los estudios bibliométricos se caracterizan porque en ellos se emplean variables que reflejan peculiaridades de los documentos. Esto permite medir de forma cuantitativa la actividad científica de cualquier medio de divulgación. Las investigaciones métricas constituyen la base para la elaboración de políticas científicas y de estudios de la ciencia. El desarrollo de la ciencia se mide por la cantidad de publicaciones de la misma. La publicación es un producto de investigación que da lugar a varios análisis.

El uso más frecuente de la bibliometría es el análisis de publicaciones seriadas. A decir de Arencibia (2012): "los estudios bibliométricos son importantes fuentes para el avance de las revistas científicas, su objeto es el tratamiento y análisis cuantitativo de las publicaciones científicas". Constituye una herramienta ampliamente usada, es un método de investigación empleado en las Ciencias de la Información para evaluar el desempeño de la investigación mediante Indicadores (Durieux \& Gevenois, 2010). A criterio de Navarrete et al. (2013) y Sánchez et al. (2015): el análisis de las publicaciones científicas constituye una herramienta indispensable para calificar la calidad del proceso de generación del conocimiento. La aplicación de este método favorece la identificación de las debilidades de las publicaciones con respecto a otras y promueve la formulación de estrategias para la mejora contínua.

Los análisis de la producción científica generalmente se apoyan de bases de datos que indizan revistas y publicaciones científicas. Varios estudios emplean indicadores bibliométricos para describir la producción científica de una temática o área específica, tal es el caso de las investigaciones desarrolladas por Cañedo et al., 2010; Murce et al., 2013; Granados et al., 2011; Franco et al., 2014; Paula et al., 2013; Peinado \& Reis, 2014; Flores \& de Andrade, 2015; Machado \& López, 2015; Zacca et al., 2015; Romero et al., 2015.

En la actualidad los análisis de citas son uno de los más empleados. A criterio de Arencibia (2008): "La idea de utilizar las referencias como términos de indización para alcanzar una mayor pertinencia en la recuperación de información científica, constituyó la base sobre la que se edificaron los tres más importantes índices de citas creados por el entonces Institute for Scientific Information de Filadelfia, Estados Unidos, actualmente bajo la denominación Thomson Scientific".

Para los análisis de citas se utilizan bases de datos que contienen las referencias de una publicación. A decir de Piedra (2010) y Melo (2015): "Los métodos basados en el uso de los indicadores biblio-ciencio-informétricos son muy utilizados pues su empleo se sustenta principalmente en el análisis de bases de datos bibliográficas". La información obtenida de las mismas tributa a la representación de los resultados de una variable. Las bases de datos pueden ser obtenidas o construidas según las características del análisis que se desea realizar.

Las referencias son un acápite importante en las comunicaciones científicas, pero a decir de Wikinski (1980), Delgado \& Martín (2015): "muchos profesionales no parecen valorar la importancia que reviste la confección del 
listado bibliográfico de su artículo escrito". Dentro de las ciencias de la información, los estudios métricos para describir la pertinencia bibliográfica tienen gran relevancia. Varios autores han utilizado las métricas para describir la actualización bibliográfica. Klenzi et al. (2012) y Macías et al. (2015) analizan métricas de similitud entre documentos de texto con el objetivo de evaluar la pertinencia de títulos bibliográficos.

Las comunidades científicas para su desarrollo requieren de fuentes bibliográficas, canales de comunicación y recursos para la investigación. Las ciencias sociales tienen menor producción científica que las ciencias técnicas. La proporción o brecha que existe entre cantidad revistas (indizadas o no) de ambas ramas del conocimiento dan fe del hecho. Otro de los indicadores que permiten contemplar las brechas entre ambas ciencias es el Índice Price.

La calidad de las publicaciones seriadas es actualmente un tema muy productivo. La endogamia editorial es uno de los aspectos más aboradados en el análisis de las revistas científicas. La endogamia editorial es la presencia del consejo científico o el comité editorial de una publicación en la autoría o las referencias. El término ha sido abordado por varios autores en investigaciones para el análisis de las publicaciones seriadas. Los autores Coslado et al. (2010) abordan el término de "endogamia evidente" que se identifica cuando a partir de datos estadísticos se observa una alta presencia del consejo científico o editorial de una publicación representado en la producción científica o las referencias. Otros autores como Cantó (2008) establecen que existe endogamia cuando se identifica el $25 \%$ o más de los miembros del comité editorial con presencia en la autoría y/o referencias. El aspecto anterior parte de la Resolución de la Comisión Nacional Evaluadora de la Actividad Investigadora (CNEAI) del año 2007 y fue ratificado por la International Journal of Psychology and Psychological Therapy en el 2010. Los postulados en relación a la endogamia editorial como forma de describir la calidad de las publicaciones para su indización, son recogidos en el estudio realizado por López (2010). Minimizar los índices de endogamia editorial, favorecerá el posicionamiento y visibilidad de las publicaciones científicas. La endogamia es actualmente analizada rigurosamente por evaluadores de fuentes de indización.

La endogamia editorial ocupa uno de los lugares principales para evaluar la calidad de las publicaciones seriadas de tipo científico. Rodríguez (2014) plantea que se deben aplicar "indicadores que pueden servir para caracterizar el comportamiento de una revista" se destacan "los referidos a la internacionalidad de los autores (...) como un indicador indirecto de la endogamia". Existen además determinados sesgos en la citación que pudieran ser indicios de endogamia. Esto está determinado por varias disciplinas científicas, donde existen autores de prestigio de obligada consulta y que a su vez estos integran el comité de expertos, revisores o editores de una publicación. Estos sesgos se describen por Bonnet et al. (2002). Otro de los sesgos que generalmente suelen cumplirse es que los autores referencien a artículos publicados por la revista a la que postulan sus manuscritos. Esto garantizará o potencialmente representa mayor posibilidad de que el artículo sea aceptado para publicar. El impacto en las revistas se mide por la cantidad de citas que reciben, por lo que publicar artículos que referencien a la revista a la que se envía el manuscrito, representa elevar su Índice H. La endogamia editorial presente en las referencias de los artículos de una publicación, no ha sido ampliamente abordada desde la literatura científica como forma de evaluar la calidad de las publicaciones. El aspecto mencionado ha sido tratado desde el empleo de otros indicadores métricos que analizan referencias. Se debe procurar identificar vías para la descripción de la endogamia en las referencias a fin de minimizarla.

La revista Centro Azúcar se encuentra hace varios años en evaluación por la base de datos SciELO. La publicación no logra entrar en el grupo de revistas indizadas por la base de datos mencionada, debido a que carece de un estudio métrico que refleje la calidad de la publicación en consonancia a los indicadores que establece SciELO. Se plantean como objetivos del estudio examinar la producción científica de la revista Centro Azúcar desde el 2011 hasta el 2015, examinar las referencias contenidas en el período mencionado e identificar los índices de endogamia editorial presentes en la productividad y las referencias de los artículos publicados por la revista. Se escoge este período de tiempo porque es el que actualmente la base de datos SciELO escogió para la última de las revisiones que efectuará en el año 2016. El estudio favorecerá a la revista presentar un conjunto de acciones encaminadas a solucionar las problemáticas que se identifiquen en la presente investigación.

\section{Metodología}

La investigación clasifica como descriptiva, longitudinal retrospectiva debido a que se su objeto de centra solo en describir la producción científica y las referencias de la revista que se analiza en el período comprendido entre los años 2011 al 2015. El estudio presenta un enfoque mixto de tipo cualitativo dominante. 


\subsection{Técnicas y fuentes}

Las técnicas empleadas que facilitan la recogida de información son la revisión de documentos para la localización de referentes teórico-prácticos sobre estudios de productividad científica y el análisis porcentual.

\subsection{Materiales y métodos}

Se emplean métodos en el nivel teórico y empírico para la recogida de los datos. En el nivel empírico se utiliza el analítico-sintético, sistémico-estructural, histórico-lógico e inductivo-deductivo. A nivel empírico se emplea el análisis documental, la triangulación de información y el método bibliométrico como método matemático dentro de las Ciencias de la Información. Los pasos a seguir en este método se describen a continuación:

- Fuentes de información: se utiliza como fuente de información primaria todos los artículos publicados por la revista Centro Azúcar en el período comprendido entre el 2011 y 2015.

- Confección de la base de datos: se registran los artículos y las referencias contenidas en los mismos, correspondientes al período mencionado. Se realiza una base de datos en el gestor bibliográfico EndNote en su versión X7. Fueron procesados un total de 180 artículos y 682 referencias pertenecientes los mismos. Se crearon las bases de datos bibliográficas: "Productividad de Centro Azúcar" y "Referencias de Centro Azúcar". Para el análisis se emplearon el mismo número de campos localizados en la base de datos realizada y se adicionó uno para la identificación del idioma.

- Normalización y procesamiento de los datos: Se normaliza la base de datos teniendo en cuenta para ello la eliminación de duplicados en los artículos y aspectos de la gramática española como diéresis, tildes y la letra ñ con la finalidad de obtener resultados fiables. Para la obtención de rankings y para el cálculo de los indicadores seleccionados se empleó el programa Microsoft Excel, del paquete de programas Microsoft Office 2007, donde fueron creadas tablas y gráficos correspondientes. La dimensión temporal del estudio abarca el período de los 5 años mencionados con anterioridad.

Indicadores escogidos: Los indicadores que se emplean son multidimensionales ya que se utilizan para medir varias características dentro del análisis métrico que se realiza: producción científica, endogamia editorial y análisis de referencias. Para la productividad como unidad de las de observación del método se emplean los indicadores que se reflejan en la Tabla 1.

\begin{tabular}{|l|l|}
\hline \multicolumn{1}{|c|}{ Indicador } & \multicolumn{1}{c|}{ Operacionalización } \\
\hline \multirow{3}{*}{ Productividad por años } & $\begin{array}{l}\text { Permitirá conocer y describir el comportamiento de la producción científica en los } \\
\text { años que se analizan. La fórmula de cálculo es la siguiente: } \mathrm{A}(\mathrm{n})=\mathrm{r} 1+\mathrm{r} 2+\mathrm{r} 3+\ldots . .\end{array}$ \\
& $\begin{array}{l}\text { Donde A es el año y r los trabajos publicados. } \\
\text { Para la visualización de los resultados se realiza un ranking y se grafican los } \\
\text { resultados atendiendo al orden cronológico de los años que se analizan en } \\
\text { consonancia a la Ley de Price. }\end{array}$ \\
\hline Productividad por idioma & $\begin{array}{l}\text { Se obtiene a partir de la elaboración de un ranking de forma descendente y refleja el } \\
\text { idioma más productivo en la publicación. }\end{array}$ \\
\hline
\end{tabular}

Tabla 1: Operacionalización de los indicadores escogidos para describir la producción científica de la revista Centro Azúcar entre los años 2011 y 2015 (fuente: elaboración propia). 


\begin{tabular}{|c|c|}
\hline Indicador & Operacionalización \\
\hline $\begin{array}{l}\text { Productividad por } \\
\text { autores }\end{array}$ & $\begin{array}{l}\text { Permitirá conocer los autores más productivos, además de facilitar datos para el } \\
\text { posterior cálculo de la endogamia de la revista. Para la obtención de datos se } \\
\text { elaborará un ranking de forma descendente. }\end{array}$ \\
\hline $\begin{array}{l}\text { Autores más productivos } \\
\text { por años }\end{array}$ & $\begin{array}{l}\text { Se establece a partir de un ranking de forma descendente y refleja los autores que } \\
\text { más producen a partir de los años de la publicación que se analiza. Para el análisis } \\
\text { de los datos se elabora una matriz que es visualizada a través de un gráfico de } \\
\text { dispersión. }\end{array}$ \\
\hline $\begin{array}{l}\text { Índice de coautoría por } \\
\text { años. }\end{array}$ & 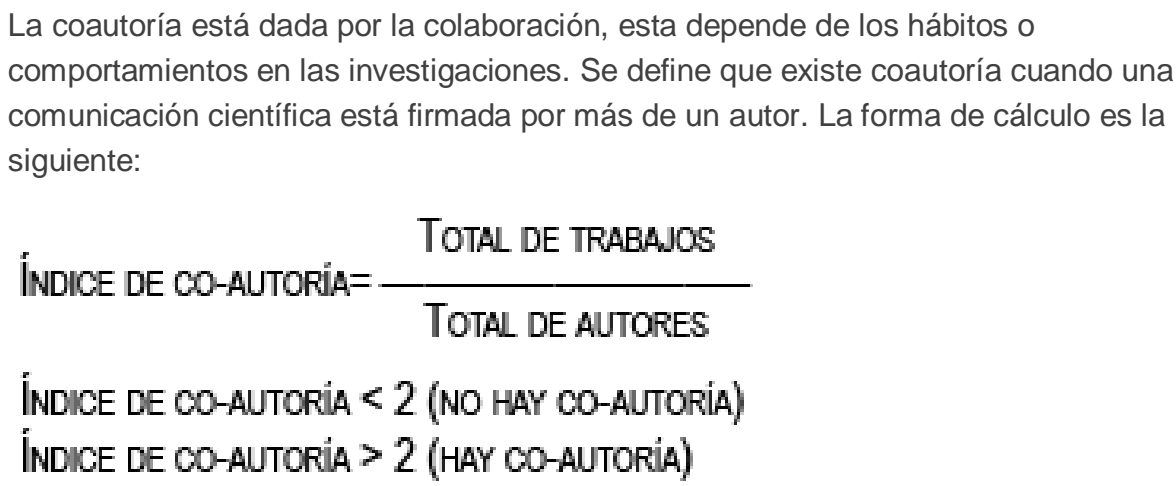 \\
\hline $\begin{array}{l}\text { Distribución de los } \\
\text { autores según su } \\
\text { productividad }\end{array}$ & $\begin{array}{l}\text { Atendiendo al nivel de productividad presentado por los autores identificados dentro } \\
\text { de la muestra, estos se distribuyeron en } 3 \text { grandes grupos: } \\
\text { Grandes Productores: Producen } 10 \text { o más artículos } \\
\text { Medianos Productores: Producen entre } 5 \text { y } 9 \text { trabajos } \\
\text { Pequeños Productores: Producen entre } 1 \text { y } 5 \text { artículos. }\end{array}$ \\
\hline Índice de Transitoriedad & $\begin{array}{l}\text { Los autores transitorios son aquellos que presentan } 1 \text { sola publicación. El índice de } \\
\text { transitoriedad se refleja a partir del porcentaje de autores con una publicación } \\
\text { atendiendo al total de autores. La forma de cálculo es la siguiente: IT= } \\
\text { (AT/Ta) }{ }^{*} 100 \% \\
\text { Donde AT: Autor transitorio y Ta: Total de autores identificados en la muestra. }\end{array}$ \\
\hline
\end{tabular}

Tabla 1: Operacionalización de los indicadores escogidos para describir la producción científica de la revista Centro Azúcar entre los años 2011 y 2015 (fuente: elaboración propia) - Continuación.

Los indicadores empleados para analizar las referencias se pueden observar en la Tabla 2.

\begin{tabular}{|l|l|}
\hline \multicolumn{1}{|c|}{ Indicador } & \multicolumn{1}{c|}{ Operacionalización } \\
\hline $\begin{array}{l}\text { Tipologías documentales } \\
\text { referenciada }\end{array}$ & $\begin{array}{l}\text { Permitirá conocer y describir la tipología de fuentes que más se emplean para la } \\
\text { confección de las referencias bibliográficas de los artículos que se publican. }\end{array}$ \\
\hline Revista más referenciada & $\begin{array}{l}\text { A partir del análisis de las instituciones productoras se podrán emitir criterios sobre } \\
\text { la presencia de endogamia editorial evidente. Esta se cumple cuando más del } 30 \% \\
\text { de las instituciones o las referencias se relacionan con la revista o el centro donde } \\
\text { se encuentra localizada. }\end{array}$ \\
\hline $\begin{array}{l}\text { Autores más } \\
\text { referenciados }\end{array}$ & $\begin{array}{l}\text { Muestra el cúmulo de autores más citados en el período analizado, siendo estos los } \\
\text { que más impacto han alcanzado dentro de los investigadores que publican en la } \\
\text { revista centro Azúcar. Para la visualización de resultados se elabora un ranking de } \\
\text { forma descendente. }\end{array}$ \\
\hline
\end{tabular}

Tabla 2: Operacionalización de los indicadores escogidos para describir la endogamia editorial entre los años 2011 y 2015 (fuente: elaboración propia). 


\begin{tabular}{|l|l|}
\hline \multicolumn{1}{|c|}{ Indicador } & \multicolumn{1}{|c|}{ Operacionalización } \\
\hline Años más referenciados & $\begin{array}{l}\text { Refleja los años con mayor presencia en los repertorios bibliográficos de la } \\
\text { publicación. Se establece a través de un ranking de forma descendente. }\end{array}$ \\
\hline $\begin{array}{l}\text { Para calcular la pertinencia de la bibliografía se emplea la siguiente fórmula aplicada } \\
\text { a cada año objeto del análisis: }\end{array}$ & $\begin{array}{l}\text { IP }=\sum \text { R } \\
\text { Índice } / \sum \text { Price por años } \\
\text { hayan sido publicadas en los últimos } 5 \text { años con respecto a la publicación del } \\
\text { artículo y su año y } \sum \text { TR: Sumatoria del total de referencias citadas en cada año o en } \\
\text { general. Para caracterizar la actualidad bibliográfica se utiliza la escala siguiente: } \\
\text { Índice de Price de } 0 \text { hasta 0,3 es bajo. } \\
\text { Índice de Price de 0,3 hasta 0,5 es bajo a medio. } \\
\text { Índice de Price de 0.5 hasta 0.7 es medio a alto. } \\
\text { Índice de Price de 0.7 hasta 1 es alto. }\end{array}$ \\
\hline $\begin{array}{l}\text { Se aplica la fórmula mencionada con anterioridad, pero para este caso se emplea } \\
\text { como objeto del estudio el total de referencias analizadas. }\end{array}$ \\
\hline
\end{tabular}

Tabla 2: Operacionalización de los indicadores escogidos para describir la endogamia editorial entre los años 2011 y 2015 (fuente: elaboración propia) - Continuación.

Los indicadores empleados para analizar la endogamia editorial se pueden observar en la Tabla 3.

\begin{tabular}{|l|l|}
\hline \multicolumn{1}{|c|}{ Indicador } & \multicolumn{1}{c|}{ Operacionalización } \\
\hline $\begin{array}{l}\text { Instituciones productoras } \\
\text { de comunicaciones } \\
\text { científicas }\end{array}$ & $\begin{array}{l}\text { Permitirá conocer y describir el comportamiento de la presencia de instituciones en } \\
\text { los artículos que se publican en la revista. Se establecerá a partir de un ranking de } \\
\text { forma descendente con la cantidad de artículos que ha publicado cada institución. }\end{array}$ \\
\hline Endogamia evidente. & $\begin{array}{l}\text { A partir del análisis de las instituciones productoras se podrán emitir criterios sobre } \\
\text { la presencia de endogamia editorial evidente. Esta se cumple cuando más del } 30 \% \\
\text { de las instituciones o las referencias se relacionan con la revista o el centro donde } \\
\text { se encuentra localizada. }\end{array}$ \\
\hline $\begin{array}{l}\text { Porcentaje de } \\
\text { endogamia }\end{array}$ & $\begin{array}{l}\text { Se realiza a partir del recuento de la cantidad de artículos cuyos autores y/o } \\
\text { referencias se relacionan con la publicación y posteriormente se dividen entre el } \\
\text { total de artículos publicados o el total de referencias respectivamente. }\end{array}$ \\
\hline
\end{tabular}

Tabla 3: Operacionalización de los indicadores escogidos para describir la endogamia editorial entre los años 2011 y 2015 (fuente: elaboración propia).

\subsection{Población y muestra:}

La muestra está constituida por el total de artículos publicados durante el 2011 y hasta diciembre del 2015. El período de tiempo seleccionado se corresponde con el análisis que realizará la base de datos SciELO en el año 2016, a partir de que en el segundo semestre de 2015 la revista fuera incluida en la colección SciELO Cuba. Se establecieron como categorías de análisis la producción científica de la revista, la endogamia editorial y las referencias contenidas en los artículos publicados. 


\section{Resultados}

\subsection{Caracterización del dominio.}

La revista Centro Azúcar publica artículos originales, revisiones y comunicaciones cortas sobre las temáticas de "tecnología, cultivo de la caña, maquinaria, energética, organización y gestión de las producciones azucareras y sus derivados. En su versión digital el alcance se amplía hacia temas relacionados con el medio ambiente y secciones misceláneas extendidas hacia energías renovables, petróleo y petroquímica" (Centro Azúcar, 2015). La revista fue fundada en 1973 y publica artículos en español sobre la producción de azúcar y otros temas afines. Es de acceso abierto, que significa que todo el contenido está disponible gratuitamente, sin cargo alguno para el usuario o su institución

La revista tiene declarada la presencia de miembros de los comités editoriales y científicos, identificados en la página web de la revista en la etiqueta de Comité Editorial. La revista es arbitrada por especialistas de gran experiencia y prestigio donde la mayoría de los profesionales cuenta con grado científico de doctor. La publicación cuenta con instrucciones detalladas a los autores, de igual forma ubicadas en la página web de la publicación en la etiqueta Información para autores.

Se ofrecen normas para los tipos de comunicaciones científicas que acepta la revista: Artículos originales: estos deben ser trabajos completos con especial importancia y deben presentarse de forma clara y concisa. Revisiones: Los autores de artículos de revisión deben consultar previamente a los editores para conocer la posible aceptación del tópico en cuestión. Comunicaciones cortas: Serán aceptadas comunicaciones cortas de investigaciones en estado incipiente y con potencial para su difusión a través de la revista (Centro Azúcar, 2015).

Se identifica la ppresencia de información sobre proceso de evaluación y selección de manuscritos empleados por la revista, editorial, comité de selección, incluyendo los criterios, procedimiento y plan de revisión de los revisores. La información referida de ubica dentro de las normas de redacción para los autores. La publicación exige la traducción a diferentes idiomas de sumario, palabras clave y resúmenes; en el caso particular de la publicación que se analiza se constata la traducción al idioma inglés de la totalidad de los títulos, palabras clave y resumen de las comunicaciones publicadas. La revista tiene una frecuencia trimestral y se constata que la misma sale en el tiempo establecido y no se ha omitido la salida de ningún número.

Para la revisión de las contribuciones se emplea el sistema de doble ciego y se declara en la página web de la publicación la exigencia del anonimato en la revisión de los originales. La situación anterior se torna un poco difícil para los editores debido a que la publicación no emplea un sistema de gestión automatizado para publicaciones seriadas. Actualmente el envío de las propuestas a la revista es por vía correo electrónico, aspecto que provoca la poca confiabilidad de los autores por el sistema de doble ciego que se emplea. En la etiqueta Información para autores, de la página web de la revista se explicita el procedimiento de la revisión a ciegas. Para el primer semestre del 2016 la publicación debe emplear el sistema automatizado para la gestión de publicaciones seriadas en línea Open Journal System (OJS), aspecto que favorecerá la indización de la publicación. Otro aspecto a considerar es la declaración de la autoría de las comunicaciones científicas que se publican en la revista, debido a que no hay uniformidad a la hora de establecer el nombre de los autores.

La comunicación motivada de la decisión editorial se declara en el sitio web de la revista. Se explica la forma de comunicar los resultados del proceso de revisión. Por su actividad la revista Centro Azúcar se relacionas con varias instituciones y centros de investigación. En la tabla 4 se mencionan los centros que han publicado en el dominio. 


\begin{tabular}{|c|c|}
\hline Centro & Siglas \\
\hline Universidad Central "Marta Abreu" de Las Villas, Cuba & UCLV \\
\hline Centro de Bioactivos Químicos & $\mathrm{CBQ}$ \\
\hline Universidad "Carlos Rafael Rodríguez" de Cienfuegos, Cuba & UCF \\
\hline Universidad "Ignacio Agramonte" de Camagüey, Cuba & UC \\
\hline Universidad "José Marti" de Sancti Spíritus, Cuba & UNISS \\
\hline Universidad "Camilo Cienfuegos" de Matanzas, Cuba & UMTZ \\
\hline Centro universitario Municipal Aguada de Pasajeros, Cuba & CUM-AGUADA \\
\hline Centro Universitario “José Antonio Echeverría”, Cuba & CUJAE \\
\hline Universidad San Carlos de Guatemala & USCG \\
\hline Universidad Nacional de Misiones, Argentina & UNMA \\
\hline Universidad Federal de Pernambuco, Brasil & UFP \\
\hline Universidad de Tolima, Colombia & UTC \\
\hline Lousiana State University Agricultural Center & LSUAC \\
\hline Consejo Profesional de Ciencias Naturales, Argentina & $\mathrm{CPCN}$ \\
\hline División de Talleres "Enrique Villegas", Santa Clara, Cuba & TALL-SC \\
\hline Empresa Planta Mecánica, Santa Clara, Cuba & PMC-SC \\
\hline Empresa Provincial de Alimentos, Santa Clara, Cuba & EMPA-SC \\
\hline Empresa CUPET, Santa Clara, Cuba & CUPET-SC \\
\hline Empresa Geominera del Centro, Santa Clara, Cuba & GEO-SC \\
\hline Unidad Empresarial "9 de Abril”, Sagua La Grande, Cuba & UEB-SAGUA \\
\hline Empresa de Soldar Carriles "Tony Santiago", Placetas, Cuba & ESC-PLACETAS \\
\hline Empresa Azucarera "Perucho Figueredo", Encrucijada, Cuba & CAI-ENCRUCIJADA \\
\hline Instituto Nacional de Investigaciones de la Caña de Azúcar & INICA \\
\hline Grupo Empresarial AZCUBA, Cienfuegos, Cuba & AZCUBA-CFG \\
\hline Refinería "Camilo Cienfuegos", Cienfuegos, Cuba & REF-CFG \\
\hline Empresa de Ingeniería y Proyectos para la Electricidad & INEL-CFG \\
\hline Grupo Empresarial AZCUBA, Las Tunas, Cuba & AZCUBA-LT \\
\hline Centro de derivados “Amancio Rodríguez", Las Tunas, Cuba & DERV-LT \\
\hline
\end{tabular}

Tabla 4: Entidades que se relacionan con el dominio y que tienen productividad científica (fuente: elaboración propia).

Las instituciones mencionadas tienen un vínculo profesional con la revista Centro Azúcar que se traduce en la publicación de artículos y aplicación de proyectos de colaboración. 


\subsection{La producción científica de la revista Centro Azúcar entre el 2011 y 2015.}

La revista Centro Azúcar presenta altos índices de coautoría, representando a más de 5 autores por artículos. Para el análisis de los resultados obtenidos en cuanto a la coautoría se realiza una distribución de la de artículos publicados por años y la cantidad de autores que estos contenían. Los resultados de la coautoría por años y total se muestran en la Tabla 5.

\begin{tabular}{|c|c|c|c|}
\hline Año & Cantidad de artículos & Cantidad de autores & Índice de coautoría \\
\hline 2011 & 40 & 192 & 4.8 \\
\hline 2012 & 31 & 177 & 5.7 \\
\hline 2013 & 34 & 159 & 4.8 \\
\hline 2014 & 37 & 147 & 3.9 \\
\hline 2015 & 38 & 149 & 3.9 \\
\hline Total & 180 & 824 & 4.57 \\
\hline
\end{tabular}

Tabla 5: Distribución de autores, títulos y coautoría por años (fuente: elaboración propia).

Existe un comportamiento medio en cuanto a la coautoría. Los valores totales sobrepasan en gran medida la cifra mínima (2), logrando cómo resultado grandes índices de coautoría. El resultado no es favorable, pues aunque los investigadores tengan una alta productividad, los altos índices de coautoría reflejan la existencia de pocos especialistas al identificarse muchos autores en los artículos. Debe destacarse que por lo general en las investigaciones sobre química aplicada los autores no publican solos, las investigaciones se desarrollan con el apoyo de técnicos y otros especialistas que desarrollan estudios prácticos en laboratorios.

Se emplearon solamente dos idiomas: el inglés y el español. El idioma más favorecido fue el espeañol con un total de 178 artículos. El resultado obtenido es algo esperado en consonancia al idioma oficial de la pulicación, a la nación donde se edita la revista y producto de la ausencia de traducciones de las normas editoriales. El idioma español representa el $98.88 \%$ del total de artículos publicados. Atendiendo a la productividad por autores se identificaron 824 autores en los 180 artículos. Los autores más productivos se observan en la Tabla 6.

\begin{tabular}{|l|c|c|c|}
\hline Autor & Cantidad de artículos & Autor Principal & Coautor \\
\hline Gonzáles-Suárez, Erenio & 37 & 2 & 35 \\
\hline Mesa-Garriga, Layanis & 12 & 3 & 9 \\
\hline Morales-Zamora, Marlén & 6 & 0 & 5 \\
\hline De León-Benítez, Juan B. & 6 & 2 & 4 \\
\hline Miño, Juan Esteban & 6 & 1 & 5 \\
\hline Gallardo-Aguilar, Irenia & 6 & 1 & 4 \\
\hline Villanueva-Ramos, Gretel & 5 & 1 & 4 \\
\hline Moya-Rodríguez, Jorge L. & 5 & 1 & 4 \\
\hline López-Bello, Nancy & 5 & 1 & 6 \\
\hline
\end{tabular}

Tabla 6: Autores más productivos (fuente: elaboración propia).

Se destaca a Gonzáles-Suárez, Erenio con un total de 37 artículos publicados, de ellos solo dos como autor principal. El autor mencionado labora dentro de la revista como editor y revisor. Lo anterior provoca que se eleve grandemente los índices de endogamia editorial en la publicación que puede traer graves consecuencias como la no aceptación de la publicación para su indización en bases de datos de prestigio. El segundo autor más 
productivo también labora dentro de la institución donde se encuentra ubicada la revista, específicamente en el $\mathrm{CBQ}$ de la UCLV. Esto aumenta la proporción de endogamia, téngase en cuenta que los autores más productivos proceden del centro donde se edita la revista. Se realiza un análisis de la productividad científica de los autores por años. Los resultados se representan en la llustración 1.

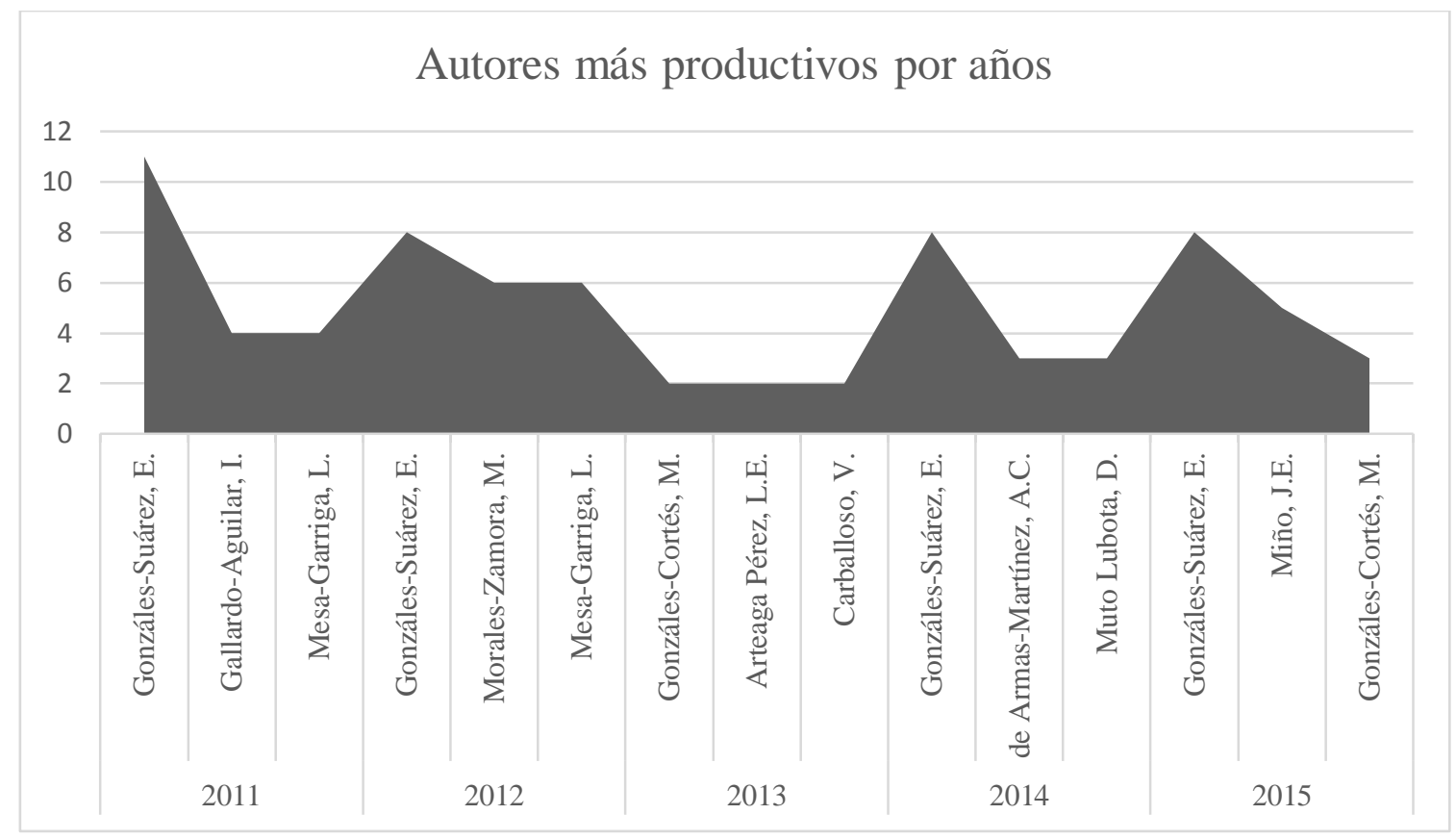

Ilustración 1: Autores más productivos por años (fuente: elaboración propia).

Se identifica un alto número de autores transitorios en correspondencia a la cantidad de artículos que se publican en los años analizados. En el año 2011 aparecen 211 autores transitorios, en los cuatro años que le siguen se identifican 192, 80, 87 y 82 autores respectivamente. Del total de 824 autores se encontraron 652 transitorios por lo que el índice de transitoriedad es de un $79.12 \%$.

Atendiendo a la producción científica de los autores, estos son clasificados a partir de su presencia en el período analizado. Se identifican como grandes productores solamente a Gonzáles-Suárez, Erenio y Mesa-Garriga, Layanis. Como medianos productores se establecen 6 autores, estos son: Morales-Zamora, Marlén, VillanuevaRamos, Gretel, De León-Benítez, Juan B., Gallardo-Aguilar, Irenia, Moya-Rodríguez, Jorge L. y López-Bello, Nancy. El resto se considera como pequeños productores de comunicaciones científicas.

\subsection{Análisis de las referencias de los artículos publicados por la revista Centro Azúcar entre el 2011 y 2015.}

Las referencias de la publicación no han sido objeto de análisis con anterioridad. La identificación de las características de las referencias de la revista favorecerá la mejora de la publicación. Las referencias son uno de los aspectos con más peso en el análisis de las publicaciones seriadas en línea. Los metadatos que se identifican en las referencias varían de una fuente a otra. De forma permanente se declara en las referencias el autor, título y año. Por la forma de la referencia el profesional de la información puede identificar la tipología documental. En las publicaciones seriadas de ciencias técnicas se deben emplear la mayor cantidad de artículos de revistas posibles con un alto grado de actualidad. La tipología documental más referenciada fueron los artículos de revista, las restantes tipologías se observan en la ilustración 2. 


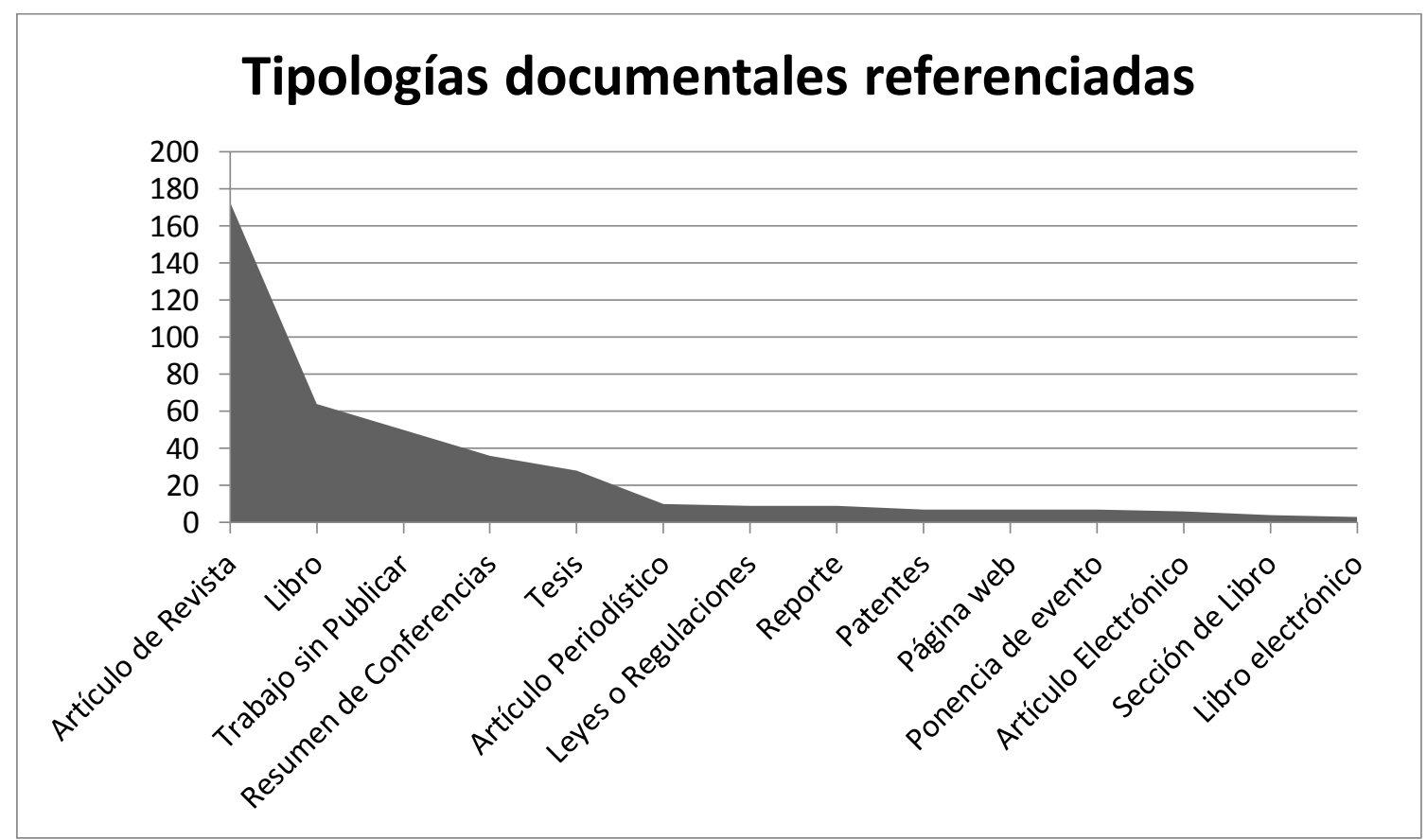

Ilustración 2: tipologías documentales referenciadas (fuente: elaboración propia).

De forma positiva los artículos de revista ocupan el primer lugar. El resultado se corresponde con las temáticas publicadas en Centro Azúcar debido a que las ciencias químicas, y las aplicadas en específico; son ciencias técnicas y por tanto tiene una mayor periodicidad de publicación y revistas científicas. Se estima que la tipología documental de artículos de revista debiera ser superior, por lo que el comité editorial debe exigir que los autores envíen no menos del $80 \%$ de las referencias de artículos de revista.

Se analiza la cantidad de referencias por años a fin de conocer de forma preliminar la actualizad de las referencias. La cantidad de referencias por años se muestran en la llustración 3.

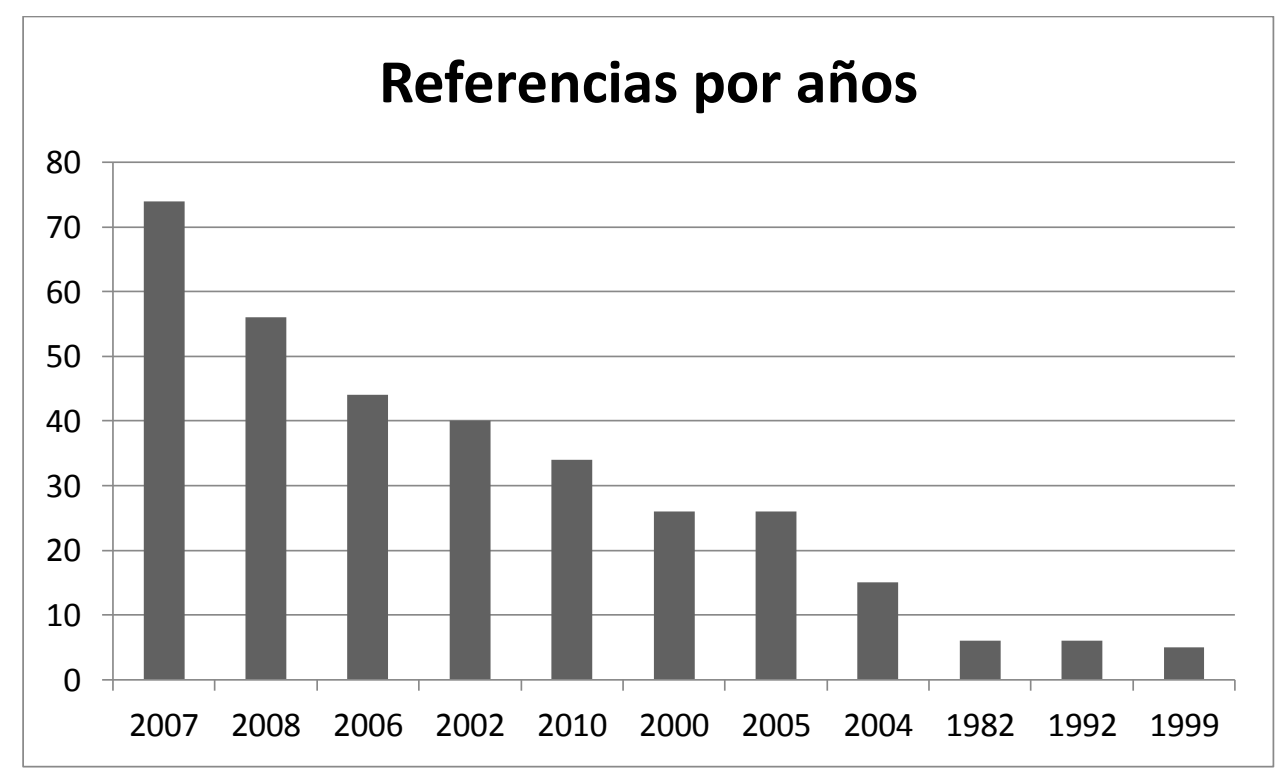

Ilustración 3: cantidad de referencias por años (fuente: elaboración propia).

Como aspecto positivo se destaca que después del año 2006 las referencias suman 298. Esta cifra se considera que debiese ser mayor por la periodicidad de publicación en las ciencias técnicas, teniendo en cuenta que las referencias anteriores al 2006 suman 384, por lo que en el análisis de la pertinencia bibliográfica se obtendrán bajos valores de índices de Price. Se analiza el comportamiento de los índices de Price por años y total, los valores se observan en la Tabla 7. 


\begin{tabular}{|c|c|c|c|}
\hline Año & Cantidad de referencias & Referencias en los últimos 5 años & Índice Price \\
\hline 2011 & 80 & 27 & 0.33 \\
\hline 2012 & 106 & 21 & 0.19 \\
\hline 2013 & 186 & 36 & 0.19 \\
\hline 2014 & 128 & 26 & 0.2 \\
\hline 2015 & 182 & 32 & 0.17 \\
\hline Total & 682 & 116 & 0.17 \\
\hline
\end{tabular}

Tabla 7: índices de Price por años y total (fuente: elaboración propia).

Los índices de Price obtenidos son bajos por lo que las referencias carecen de actualidad. Se considera con un aspecto negativo debido a que las ciencias técnicas en general y las químicas en específico poseen múltiples fuentes de información periódicas con un alto número de publicaciones. Las referencias debiesen tener menores valores que los identificados. El comité editorial de la revista deberá exigir a los autores un repertorio bibliográfico que contenga al menos, del $80 \%$ de las referencias que estén comprendidas en los últimos 5 años en el momento que se elabora la comunicación.

Los artículos de revista fueron los más citados en el período analizado. Para la identificación de las fuentes más referenciadas se elabora un ranquin de revistas, las mismas se representan en la llustración 4.

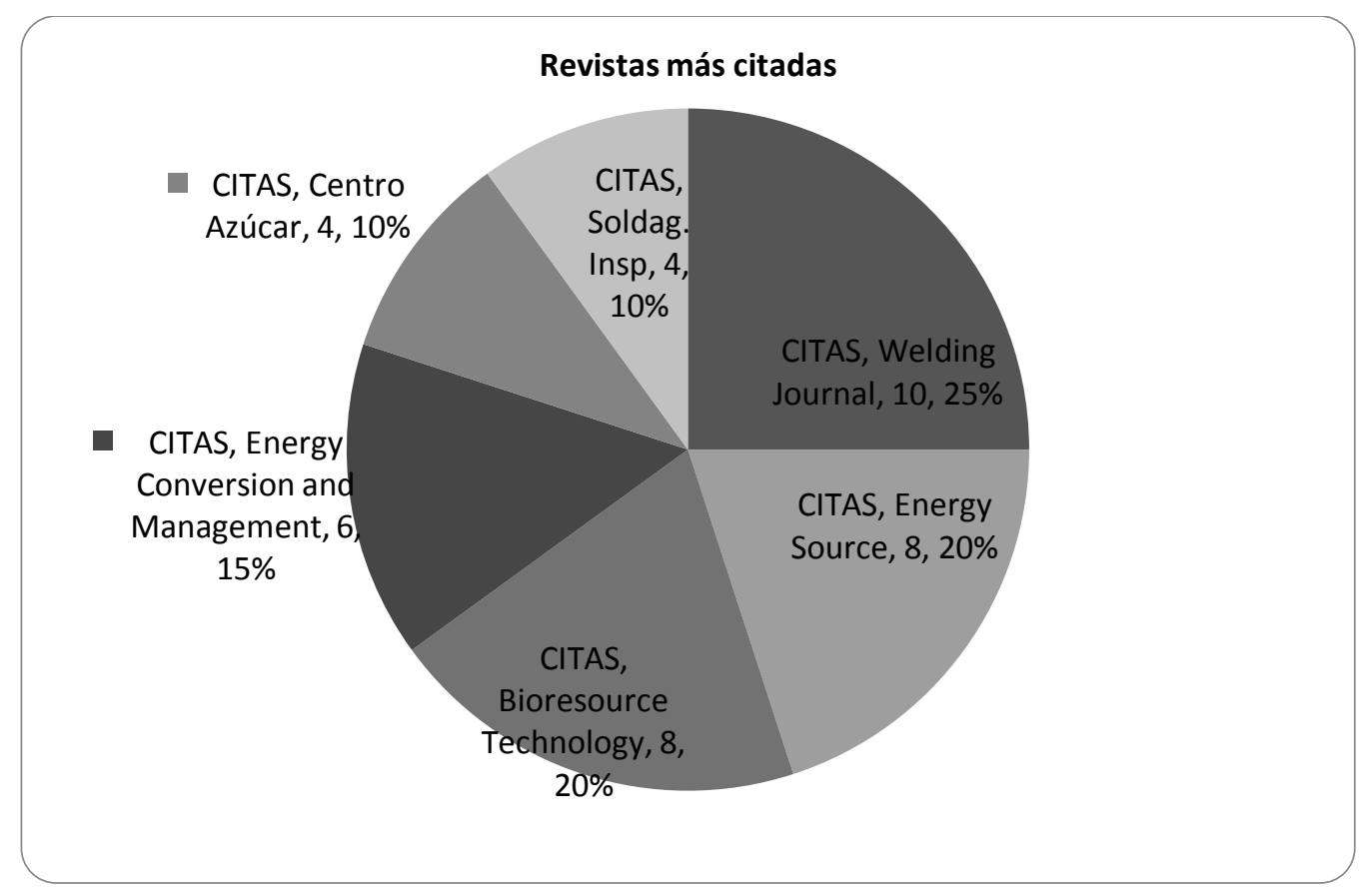

Illustración 4: revistas más citadas (fuente: elaboración propia).

Dentro de las publicaciones más referenciadas se identifica que la revista Centro Azúcar ocupa el $10 \%$ de los artículos de revista referenciados. Aunque el por ciento no es suficiente para declarar una endogamia evidente, se estima que se debe procurar disminuir la cantidad de citas que remitan a la revista donde se publica. El comité editorial debe controlar las autocitas que hagan referencia a la revista. Se analizan los autores más referenciados a fin de identificarlos que mayor impacto han tenido dentro de la publicación. Los autores más referenciados se muestran en la llustración 5 . 


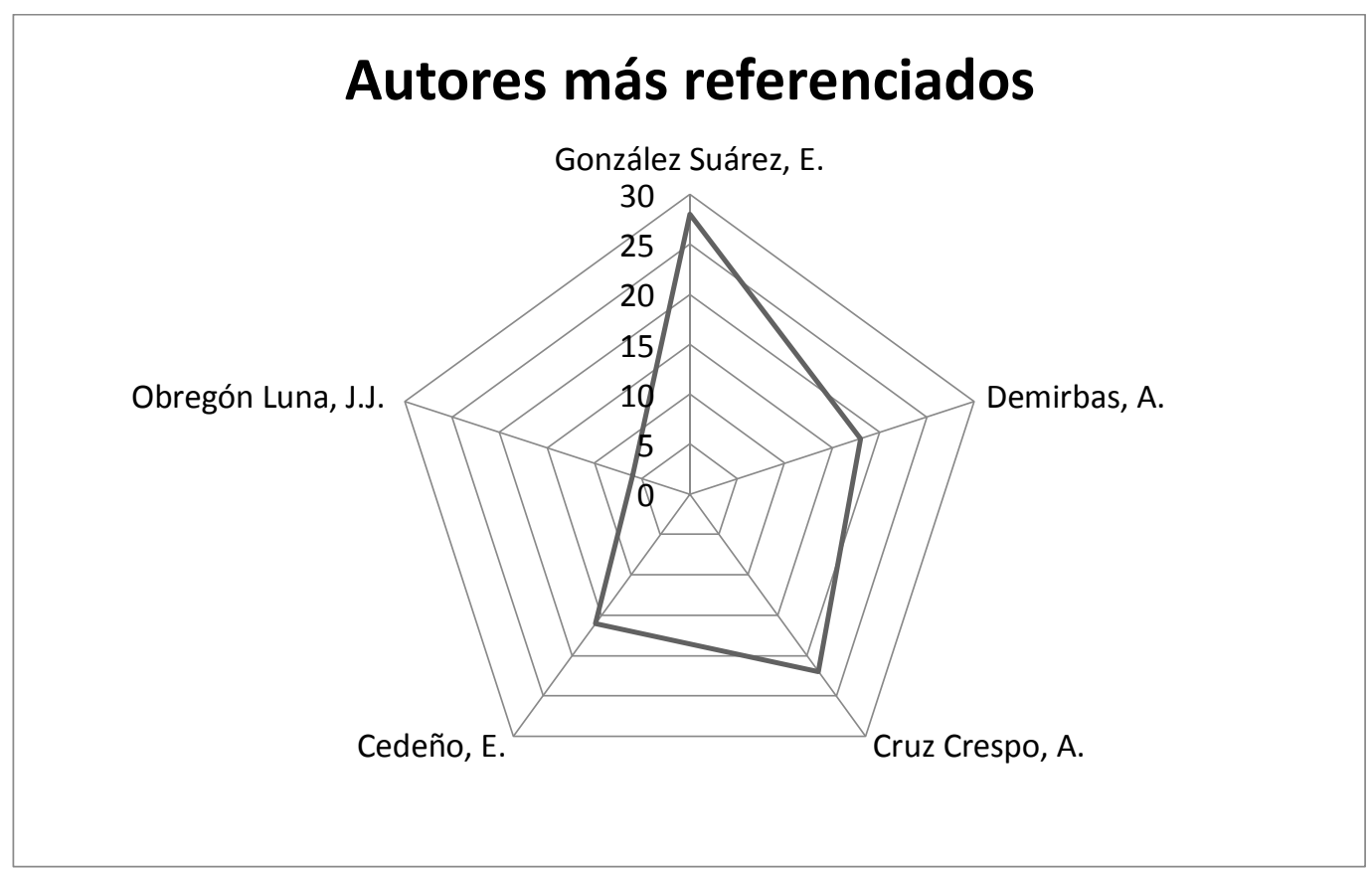

Ilustración 5: autores más referenciados (fuente: elaboración propia).

Se identifica a González Suárez, Erenio como el de mayor cantidad de citas. Este resultado refuerza los argumentos mencionados con anterioridad. El autor más citado cuenta con 28 referencias y es además miembro del comité editorial de la revista Centro Azúcar. Se realiza un análisis de los artículos producidos por el autor y se identifican autocitas descontroladas que afectan la calidad de la revista.

\subsection{Comportamiento de la endogamia editorial en la revista Centro Azúcar entre el 2011 y 2015}

Para el análisis del comportamiento de la endogamia editorial en la autoría se analizan las instituciones productoras de comunicaciones científicas. Las instituciones productoras en el período que se analiza se observan en la Tabla 8.180

\begin{tabular}{|c|c|}
\hline Institución & Cantidad de artículos \\
\hline UCLV & 113 \\
\hline UCF & 16 \\
\hline UC & 7 \\
\hline AZCUBA-LT & 5 \\
\hline UNMA & 4 \\
\hline UNISS & 3 \\
\hline INICA & 3 \\
\hline USCG & 3 \\
\hline UMTZ & 3 \\
\hline UFP & 2 \\
\hline UTC & 2 \\
\hline
\end{tabular}

Tabla 8: Autores más productivos (fuente: elaboración propia). 


\begin{tabular}{|c|c|}
\hline Institución & Cantidad de artículos \\
\hline DERV-LT & 2 \\
\hline TALL-SC & 2 \\
\hline CUJAE & 1 \\
\hline PMC-SC & 1 \\
\hline LSUAC & 1 \\
\hline CUPET-SC & 1 \\
\hline UEB-SAGUA & 1 \\
\hline ESC-PLACETAS & 1 \\
\hline GEO-SC & 1 \\
\hline EMPA-SC & 1 \\
\hline CAI-ENCRUCIJADA & 1 \\
\hline CUM-AGUADA & 1 \\
\hline UNMA & 1 \\
\hline INEL-CFG & 1 \\
\hline REF-CFG & 1 \\
\hline AZCUBA-CFG & 1 \\
\hline
\end{tabular}

Tabla 8: Autores más productivos (fuente: elaboración propia) - Continuación.

Ante los datos que reflejan las instituciones productoras de comunicaciones científicas se observa una endogamia evidente, la mayor parte de los artículos publicados proceden de la UCV, lugar donde se edita la revista. La cantidad de autores externos a la UCLV suman 67 por lo que representan el $37.22 \%$ de los autores y un 62.78 corresponde a la UCLV. Los resultados obtenidos se corresponden además con el análisis de los autores más productivos: Gonzáles-Suárez, Erenio y Mesa Garriga, Leyanis. Ambos autores son de la UCLV y colaboran directamente con la revista. Dentro de la UCLV existen varias áreas que sus investigaciones tributan a la publicación de artículos científicos en la revista Centro Azúcar: Departamento de Licenciatura en Química, Departamento de Ingeniería Química, Departamento de Licenciatura en Farmacia, CBQ, Instituto de Biotecnología de las Plantas (IBP), Departamento de Licenciatura en Biología, Departamento de Ingeniería Industrial, entre otras áreas. El comité editorial de la revista debe velar porque la publicación controle la divulgación de artículos de la institución donde se encuentra para bajar los índices de endogamia. El autor Gonzáles-Suárez, Erenio tiene 5 artículos publicados en el primer número del año 2014, cuestión negativa para la publicación.

La endogamia en las referencias se identifica en los autores más referenciados y en las revistas más referenciadas. Centro Azúcar ocupa el 10\% de los artículos de revista referenciados y se identifica a González Suárez, Erenio cuenta con la mayor cantidad de citas (28).

\section{Conclusiones}

La revista Centro Azúcar cuenta con una productividad científica caracterizada por una amplia presencia de coautores y miembros del consejo editorial/científico de la publicación. El aspecto mencionado con anterioridad afecta la calidad científica de la publicación, condicionado por la presencia de endogamia evidente y la ausencia de autores expertos.

Las referencias de los artículos publicados por la revista centro Azúcar reflejan gran cantidad de autocitas, bajos índices de Price y la presencia de insuficiente empleo de artículos de revista como tipología documental 
empleada en la elaboración de las referencias. Se identifican autores citados en las referencias que pertenecen al consejo editorial/científico de la publicación, influyendo en los índices de endogamia.

Las descripciones sobre productividad, referencias bibliográficas y endogamia editorial; contribuyen a la formulación de políticas científicas para favorecer la calidad de las publicaciones. La endogamia editorial ha sido abordada por la literatura desde el análisis de la producción científica de las publicaciones, sin embargo, se pueden establecer análisis de endogamia que partan de análisis de referencias donde se identifiquen las citas y autocitas de las publicaciones.

En el caso expuesto se muestran formas de describir la endogamia contenida en las referencias de la publicación. Se cumplen varios sesgos en la construcción de las referencias de los artículos publicados por la revista Centro Azúcar, entre ellos el de citar autores de una misma institución y a la misma publicación. Las cocitaciones favorecen que los autores aumenten su impacto, sin embargo cuando se hacen desde una publicación hacia la misma, produce aspectos negativos que atentan y hacen cuestionable el impacto que alcanza la publicación científica.

Debe potenciarse el empleo el empleo de indicadores que favorezcan la descripción de la calidad de las comunicaciones científicas de una publicación. La reformulación de políticas científicas acorde a los resultados obtenidos y en consonancia a los estándares internacionales, favorecerá que la publicación analizada sea indizada en importantes bases de datos de forma rápida.

\section{Referências}

Arencibia, R.; Moya, F.D. (2008). La evaluación de la investigación científica: una aproximación desde la perspectiva cienciométrica, Acimed, 17(4), 1-16.

Arencibia, D.F.; Betancourt, V.; González, M.; Bermello, R.; Mesa, M.E. (2012). Estudio bibliométrico de la Revista de Ciencias Médicas de La Habana en el período 2005-2009, Acimed, 4(23), 1-5.

Bemabeu, J.; Ureña, T.; Esplugues, E.M.; Trescastro, E.; Galiana, E.; Castelló, I. (2012). Las ciencias de la nutrición en la España de la segunda mitad del siglo XX; estudio bibliométrico descriptivo de la revista Anales de Bromatología (1949-1993), Nutr Hosp, 2(27), 18-25.

Bonnet, X.; Shine, R.; Lourdais, O. (2002). Taxonomic chauvinism. Trends in Ecology and Evolution, 17(2002), 1-3.

Cantó, R. (2008). Acerca de la calidad científica de la revista y el control de la endogamia RICYDE. Revista Internacional de Ciencias del Deporte, 4(10): 1-2.

Cañedo, R.; Cruz, J. (2012). Nuevos indicadores métricos para la evaluación de las publicaciones seriadas científicas y académicas. Acimed, 23(1), 84- 92.

Centro Azúcar (2015). Bienvenidos a la revista Centro Azúcar. Recuperado el 12 de septiembre de 2015 de http://centroazucar.qf.uclv.edu.cu/

Coslado, A.; Báez, J.M.; Lacunza, I. (2010). Descripción y análisis del proceso de evaluación de la calidad de las revistas científicas españolas llevado a cabo por FECYT en el año 2008. Revista Española de Documentación Científica, 33(3), 481 495.

Delgado, E.; Martín, A. (2015). Thomson Reuters y las altmetrics: usage counts frente a citation counts en la Web of Science. EC3's Document Series, 20(2015), 1-11.

Durieux, V.; Gevenois, P.A. (2010). Bibliometric indicators: quality measurements of scientific publication. Radiology, 2(2010), 342-351.

Flores, V.; de Andrade, T. (2015). Comportamento de cidadania organizacional: caracterização da produção científica internacional no período de 2002 a 2012". REV. ADM. MACKENZIE, 2(16), 45-71.

Franco, A.M.; Sanz, J.; Wanden, J.; Melian, L. (2014). La producción científica iberoamericana en ciencias de la nutrición: La indización en PubMed y Google Scholar. Nutr Hosp, 5(30), 1165-1172.

García, F.; Úbeda, M.; Marco, B. (2016). The intellectual structure of research in hospitality management: A literature review using bibliometric methods of the journal International Journal of Hospitality Management. International Journal of Hospitality Management, 52(2016), 121-130. 
Granados, M.R., Ariza, T., Gómez-García, A., y Ramiro, M.T. (2011): Estudio bibliométrico de Aula Abierta. Aula Abierta, 39 (3), 97-110.

González, F.; Osca, J. (2016). Análisis de las publicaciones españolas en la categoría Psychology Educational de la Web of Science durante el periodo 2004-2013. Aula Abierta, 44(1), 46-54.

Herculano, L. (2015). Perfil da produção científica do programa de pós-graduação em ciência da informação da Universidade de Brasília: análise dos trabalhos de enancib. Biblionline, 11(1), 89-101

Klenzi, R.; Gutierrez, L.; Villafañe, V. (2012). Técnicas de recuperación de información en la determinación de pertinencias bibliográficas. En: XIV Workshop de Investigadores en Ciencias de la Computación, Ciudad de México: México.

López, M. (2010). Estudio cuantitativo de los procesos de comunicación de Revista Latina de Comunicación Social (RLCS), 1998-2009. Revista Latina de Comunicación Social, 65(2010), 538-552.

Macías, M.E.; Rivero, M.E.; Cabrera, J.L. (2015). Proceso de evaluación de las publicaciones científicas seriadas a través de indicadores. Rev Hum Med, 15(3), 440-451.

Machado, M.O.; López, J. Producción científica relativa a los servicios de Información de medicamentos en la Web of Science. Medicent Electrón, 2(19), 63-71.

Melo, H.C. (2015). Quinze anos de estudo da revista de administração contemporânea sob a ótica da bibliometria e da rede social. Perspectivas em Gestão \& Conhecimento, 5(2015), 86-108.

Miguel, S.; Dimitri, P.J. (2013). La investigación en bibliometría en la argentina: quiénes son y qué producen los autores argentinos que realizan estudios bibliométricos. Información, Cultura y Sociedad, 29(2013), 117-138.

Murce, P.P.; Coelho, F.A.; Rezende, R.; Paschoal, T.; da Silva, A.I. (2013). A produção científica brasileira sobre a gestão de recursos humanos entre 2001 e 2010. REV. ADM. MACKENZIE, 4(15), 110-134.

Navarrete, S.; Gómez, A.; Riebeling, C.; López, G. A.; Nava, A. (2013). La investigación sobre calidad de la atención en el Instituto Mexicano del Seguro Social. Estudio bibliométrico. Salud pública de México, 6(55), 564-561.

Olmeda, C.; de Moya, F. (2016). Publishing Trends in Library and Information Sciences Across European Countries and Institutions. The Journal of Academic Librarianship, 42(1), 27-37.

Paula, C.C.; Cabrai, I.E.; Souza, L.E.O.; Brum, C. N., Silva; C. B.; Padoin, S.M.M. (2013). HIV/AIDS in childhood and adolescence. Trends in Brazilian cientific production. Invest Educ Enferm, 2(31), 277-286.

Paz, L.E.; Hernández, E.A. (2015). Estudio de productividad científica internacional de la temática Caña de Azúcar relacionada con Química Aplicada. Revista Tecnología Química, 35(3), 295-307.

Peinado, J.; Reis, A. (2014). A produção científica em gestão de operações no brasil: uma análise de temas, autores e instituições de pesquisa no período entre 2001 e 2010. REV. ADM. MACKENZIE, 5(15), 224-255.

Piedra, Y. (2010). Campo científico de la Comunicación: examinando su estructura intelectual a través del análisis de cocitación. Revista Latina de Comunicación Social, 65 (2010), 204-213.

Rodríguez, L. (2014). Indicadores bibliométricos sobre revistas: más allá de los índices de citas. En: XI Seminario HispanoMexicano de Investigación en Bibliotecología y Documentación: La información y sus contextos en el cambio social. Ciudad de México: México.

Romero, E.; Vaughan, L.; Rodriguez, L. (2015). El empleo de la Webmetría para el análisis de los indicadores de desempeño y posición financiera de la empresa: un análisis exploratorio en diversos sectores económicos de los Estados Unidos. Innovar, 25(56), 99-112.

Sánchez, A.; Carrillo, O.; Garrido, P. (2015). Análisis bibliométrico de la Revista Mexicana de Sociología basado en indicadores de citación. Revista mexicana de sociología, 77(1), 45-70.

Wikinski, J.A. (1980). Las Referencias Bibliográficas en la Evaluación del Trabajo Científico. Rev, Col. Anest, 8(188), 188-190.

Zacca, G.; Chinchilla, Z.; de Moya, F. (2015). Patrones de comunicación e impacto de la producción científica cubana en salud pública. Revista Cubana de Salud Pública, 2(41): 200-216. 


\section{Datos de los autores}

\section{Luis Ernesto Paz Enrique}

Licenciado en Ciencias de la Información. Especialista en Gestión Documental. Dirección de Recursos Humanos. Universidad Central "Marta Abreu" de Las Villas

luisernestope@uclv.cu

\section{Alejandro Céspedes Villegas \\ Licenciado en Ciencias de la Información. Profesor del Departamento de Ciencias de la Información. Facultad de Matemática, Física, Computación. Universidad Central "Marta Abreu" de Las Villas \\ acespedes@uclv.cu}

\section{Eduardo Alejandro Hernández Alfonso}

Licenciado en Comunicación Social. Profesor del Departamento de Extensión Universitaria. Dirección de Extensión, Comunicación e Informatización. Universidad Central "Marta Abreu" de Las Villas ealejandro@uclv.cu

Recibido - Received: 2015-11-09

Aceptado - Accepted: 2016-02-19

\section{$(\mathrm{cc}) \mathrm{EY}$}

This work is licensed under a Creative Commons Attribution 4.0

United States License.

\section{ULIS D-Sent}

This journal is published by the University Library System of the University of Pittsburgh as part of its D-Scribe Digital Publishing Program and is cosponsored by the University of Pittsburgh Press. 